\title{
Combination of Intravitreal Ranibizumab and Laser Photocoagulation for Aggressive Posterior Retinopathy of Prematurity
}

\author{
Ágata Mota ${ }^{a}$ Ângela Carneiro ${ }^{a, b} \quad$ Jorge Breda ${ }^{a}$ \\ Vitor Rosas $^{a} \quad$ Augusto Magalhães $^{a} \quad$ Renato Silva $^{a, b}$ \\ Fernando Falcão-Reis ${ }^{\mathrm{a}, \mathrm{b}}$ \\ ${ }^{a}$ Department of Ophthalmology, Hospital S. João, and ${ }^{b}$ University of Porto, \\ Porto, Portugal
}

\section{Key Words}

Retinopathy of prematurity - Laser photocoagulation - Ranibizumab · Anti-VEGF therapy

\begin{abstract}
Purpose: To report on 2 cases of aggressive posterior retinopathy of prematurity (ROP) treated with intravitreal ranibizumab (Lucentis ${ }^{\circledR}$ ) and laser photocoagulation.

Methods: Two premature females, born at 25 and 26 weeks' gestation with a birth weight of 530 and $550 \mathrm{~g}$, respectively, with aggressive posterior ROP received combined treatment with laser photocoagulation and intravitreal ranibizumab $(0.3 \mathrm{mg}[30 \mu \mathrm{l}])$ to each eye. Structural outcomes were evaluated by indirect ophthalmoscopy and documented by retinography.

Results: An intravitreal injection was made at 34 weeks of postmenstrual age in the first case, followed by laser photocoagulation 1 week later. There was a partial regression of ROP with treatment. Five weeks later, neovascularization regrowth with bleeding in both eyes (intraretinal and subhyaloid) occurred and retreatment with combined therapy was performed. In the second case, single therapy with laser photocoagulation was made at 34 weeks of postmenstrual age. In spite of the confluent photocoagulation in the avascular area, progression to 4A ROP stage occurred 1 week later. Both eyes were retreated 1 week later with intravitreal ranibizumab and laser photocoagulation. Treatment resulted in ROP regression in both cases. There were no signs of systemic or ocular adverse side effects. Conclusion: The cases presented show that combination therapy of indirect laser photocoagulation and intravitreal ranibizumab can be effective in the management of
\end{abstract}


aggressive posterior ROP. Further investigation on anti-VEGF safety in premature infants is necessary . Additional studies are needed to define the role of anti-VEGF in ROP treatment.

\section{Introduction}

Retinopathy of prematurity (ROP) is a proliferative disease that affects infants of low birth weight and young gestational age [1]. Both CRYO-ROP [2] and Early Treatment ROP [3] demonstrated improvements in the structural and functional outcomes with peripheral ablation therapy.

The role of VEGF in the pathogenesis of ROP has been described [4]. Many recent reports of anti-VEGF use in ROP showed that it can be a safe and effective treatment [48]. However, there is concern about the choice of the drug, dose and time of injection as well as about local and systemic potential complications [4].

Aggressive posterior ROP (APROP) is a rapidly progressing and severe form of ROP that, if untreated, progresses to stage 5. Eyes with APROP have a worse prognosis and a higher rate of retinal detachment [1].

The present report describes 2 cases of APROP treated with combined therapy including intravitreal ranibizumab (Lucentis ${ }^{\circledR}$ ) and laser photocoagulation.

\section{Methods}

ROP classification was defined according to The International Classification of Retinopathy of Prematurity Revisited [9]. APROP was characterized as stage 3 zone I with plus or preplus disease. The off-label use of anti-VEGF and the potential risks were discussed with the parents and written informed consent was obtained. All procedures were made under general anesthesia. Disinfection with povidone-iodine was made before the injections. Sterile gloves, speculum and forceps were used to perform the injections. Sterile unit doses of ranibizumab $(0.3 \mathrm{mg}$ [30 $\mu \mathrm{l}])$ were injected using a 30 -gauge needle, placed $2.5 \mathrm{~mm}$ posterior to the limbus. Immediately after the injection, and every $3 \mathrm{~h}$ for the next 7 days, topical ofloxacin was administered. Indirect ophthalmoscopy was used to look for any injury after the injections. Laser photocoagulation was applied to the $360^{\circ}$ avascular retina, extending to the ora serrata with confluent impacts. The systemic condition was closely monitored in the neonatal intensive care unit. Both cases were photographed using RetCam 3 (Clarity Medical Systems, Pleasanton, Calif., USA). Follow-up was made weekly during the first month and then monthly until total regression of ROP.

\section{Results}

The first case was a female premature infant born at 25 weeks of gestation with a birth weight of $530 \mathrm{~g}$. Her first screening examination was at 33 weeks of postmenstrual age, showing bilateral stage 3 zone I ROP with plus disease, intraretinal hemorrhages and extensive subhyaloid hemorrhage in the left eye (fig. 1 ). There was also bilateral tunica vasculosa lentis and pupillary rigidity. Ranibizumab was injected in both eyes $72 \mathrm{~h}$ after observation. One week after the treatment, there was complete regression of anterior segment vessels, reduction in central vascular dilation and tortuosity and decrease of hemorrhagic collections. Laser photocoagulation was made 1 
week after the injection. Four weeks later, there was a new subhyaloid hemorrhage in the left eye and maintenance of some intraretinal hemorrhages in the right eye. It was decided to make a second injection of ranibizumab and to increase laser photoablation in both eyes (fig. 2). Total regression of neovascularization was observed 1 week later in the right and 4 weeks later in the left eye.

The second case was a female premature infant born at 26 weeks of gestation with a birth weight of $550 \mathrm{~g}$. Her first screening examination was at 33 weeks of postmenstrual age, showing bilateral stage 3 zone I ROP with plus disease and intraretinal hemorrhages. Laser photocoagulation was made $72 \mathrm{~h}$ after observation. One week after the treatment, there was progression of the disease to stage $4 \mathrm{~A}$ and extrafoveal partial retinal detachment occurred (fig. 3). Ranibizumab injection and densification of laser photocoagulation in the avascular area were made bilaterally. Two weeks after combined treatment, regression of neovascularization could be observed (ig. 4 ).

\section{Discussion}

There are multiple reports using anti-VEGF for ROP treatment. Micieli et al. [6] showed some variability in the off-label use of bevacizumab, mainly regarding dose, time of injection and frequency of administration. ROP is less likely than choroidal neovascularization or diabetic retinopathy to necessitate repeated injections since the disease is known to undergo spontaneous involution in $90 \%$ of patients after 44 weeks of postmenstrual age [10]. The vascularization of the preterm peripheral retina is a finite process. Once the peripheral inner retinal vessels develop, the disease process does not recur [4]. Potential benefits of using anti-VEGF treatment include eliminating visual field loss due to retinal laser ablation. Injections may be administered in very sick infants to avoid general anesthesia risks, and they are easy to perform even in infants with opaque media because of anterior segment involvement [4]. Complications can occur with intravitreal injections. Jang et al. [11] reported on a patient with bilateral zone I stage 3 plus ROP who was treated with combined laser photocoagulation and ranibizumab and showed full regression 3 months after treatment but then developed bilateral retinal detachment. Honda et al. [12] reported on a patient with acute contraction of the proliferative membrane that resulted in funnel-like retinal detachment after intravitreal bevacizumab for stage 4A ROP. In recent reports, anti-VEGF was used as first-line therapy with complete regression of neovascularization without the need for further treatment [5]. Mintz-Hittner and Kuffel [4] reported that intravitreal injection is a safe and effective treatment for stage 3 ROP in zone I and II. No systemic complications have been reported to date. Some authors consider that long-term systemic complications are unlikely following a single injection of a small quantity of anti-VEGF into the vitreous cavity [7]. We used ranibizumab because it has a shorter systemic half-life than bevacizumab [13-15], which in our opinion may reduce the risk of systemic complications in premature infants. On the other hand, the shorter half-life in vitreous humor may explain the need for reintervention in the first case. Several investigators have confirmed the presence of bevacizumab in the serum after an intravitreal injection, which lasted for 1-2 weeks. Also, there are reports of dramatic reductions in the systemic VEGF levels following intravitreal bevacizumab, which seems to continue for several days [15]. 
This is the second report using ranibizumab as ROP treatment. There were no signs of systemic or ocular adverse side effects until the time of this report. The combination therapy was effective in the management of APROP. Further investigation on anti-VEGF safety in ROP treatment is necessary.

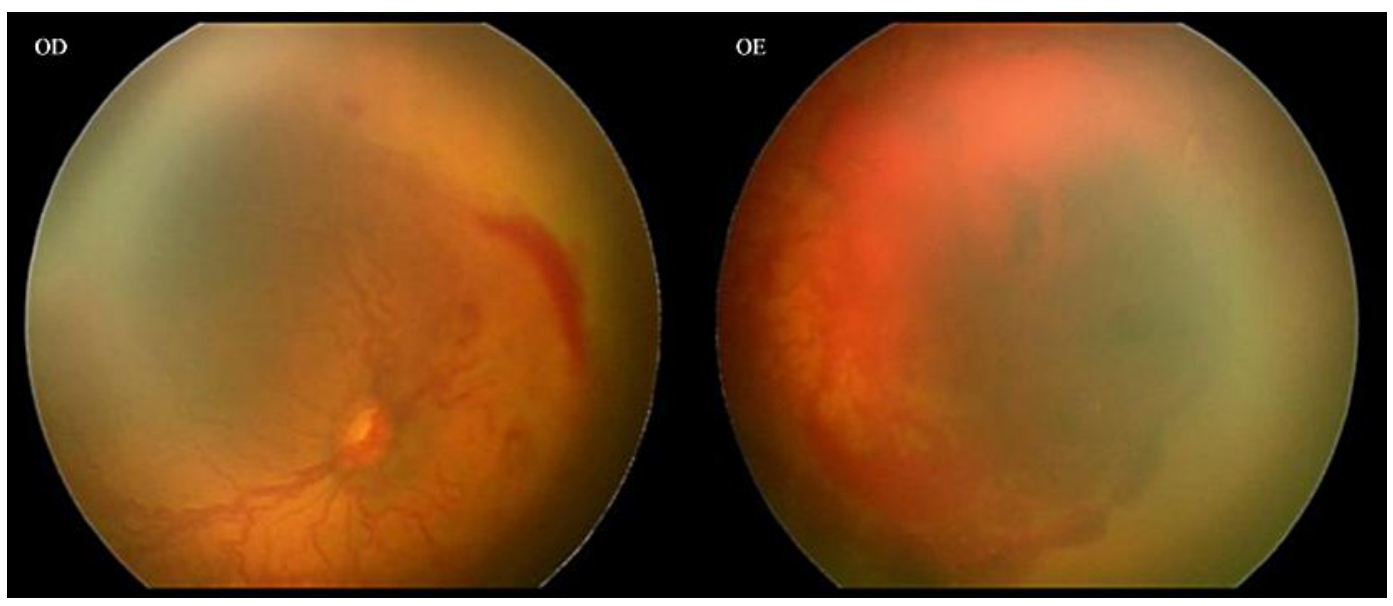

Fig. 1. Bilateral stage 3 zone I ROP with plus disease, intraretinal hemorrhages and extensive subhyaloid hemorrhage in the left eye.

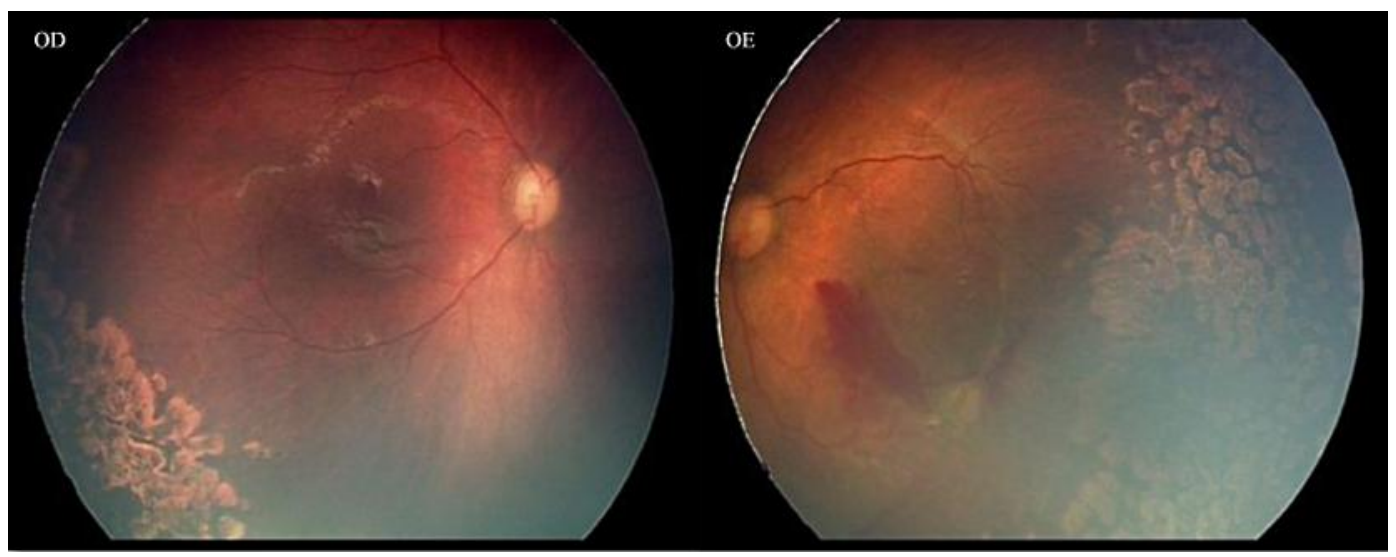

Fig. 2. Total regression of neovascularization could be observed in both eyes after the treatment. 


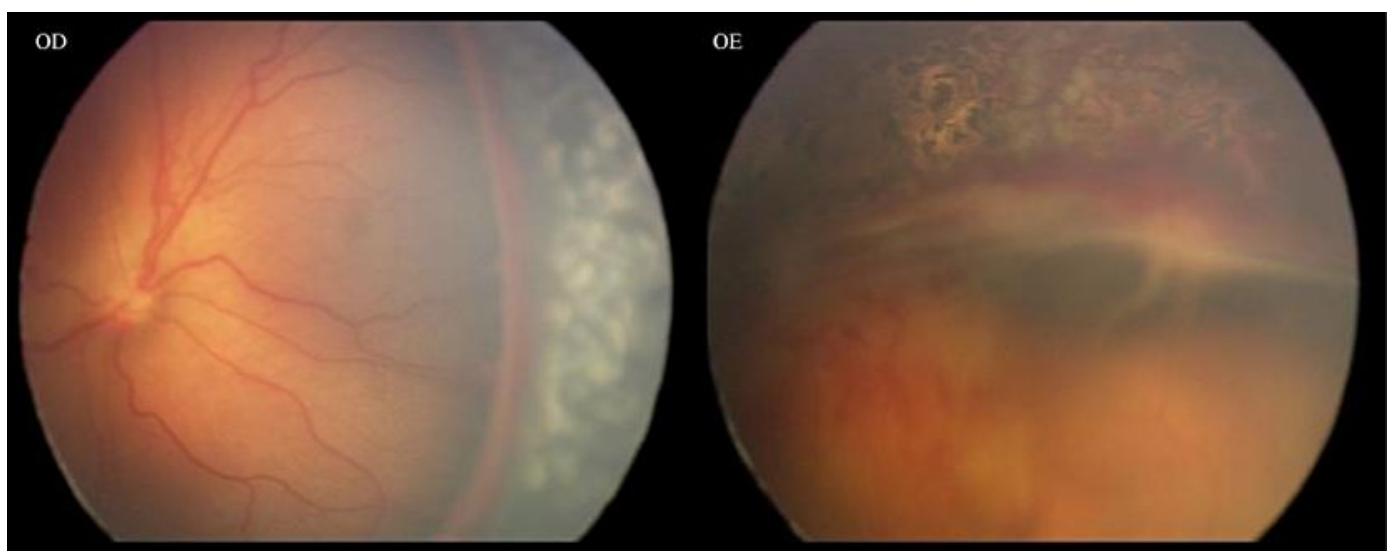

Fig. 3. One week after laser treatment, progression of the disease to stage $4 \mathrm{~A}$ could be observed in the left eye.

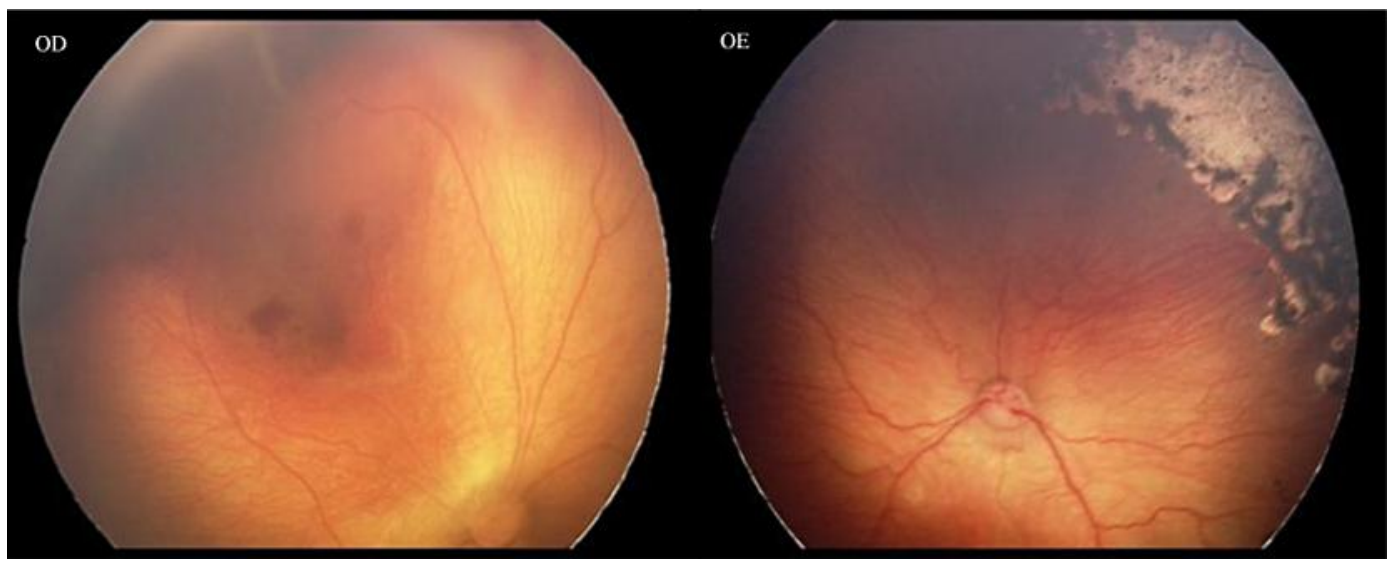

Fig. 4. Two weeks after combined treatment, regression of neovascularization could be observed.

\section{References}

1 Drenser KA, Trese MT, Capone A: Aggressive posterior retinopathy of prematurity. Retina 2010;30:S37S40.

2 Cryotherapy for Retinopathy of Prematurity Cooperative Group: Multicenter trial of cryotherapy for retinopathy of prematurity. Preliminary results. Arch Ophthalmol 1988;106:471-479.

-3 Vander JF, McNamara JA, Tasman W, Brown GC: Revised indications for early treatment of retinopathy of prematurity. Arch Ophthalmol 2005;123:406-407.

4 Mintz-Hittner HA, Kuffel RR: Intravitreal injection of bevacizumab (Avastin) for treatment of stage 3 retinopathy of prematurity in zone I or posterior zone II. Retina 2008;28:831-838.

5 Dorta P, Kychenthal A: Treatment of type 1 retinopathy of prematurity with intravitreal bevacizumab (Avastin). Retina 2010;30:S24-S31.

6 Micieli JA, Surkont M, Smith AF: A systematic analysis of the off-label use of bevacizumab for severe retinopathy of prematurity. Am J Ophthalmol 2009;148:536-543.e2.

7 Mintz-Hittner HA, Best LM: Antivascular endothelial growth factor for retinopathy of prematurity. Curr Opin Pediatr 2009;21:182-187. 
8 Kong L, Mintz-Hittner HA, Penland RL, Kretzer FL, Chévez-Barrios P: Intravitreous bevacizumab as antivascular endothelial growth factor therapy for retinopathy of prematurity: a morphologic study. Arch Ophthalmol 2008;126:1161-1163.

$>9$ International Committee for the Classification of Retinopathy of Prematurity: The International Classification of Retinopathy of Prematurity revisited. Arch Ophthalmol 2005;123:991-999.

$\$ 10$ Chung EJ, Kim JH, Ahn HS, Koh HJ: Combination of laser photocoagulation and intravitreal bevacizumab (Avastin) for aggressive zone I retinopathy of prematurity. Graefes Arch Clin Exp Ophthalmol 2007;245:1727-1730.

11 Jang SY, Choi KS, Lee SJ: Delayed-onset retinal detachment after an intravitreal injection of ranibizumab for zone 1 plus retinopathy of prematurity. J AAPOS 2010;14:457-459.

-12 Honda S, Hirabayashi H, Tsukahara Y, Negi A: Acute contraction of the proliferative membrane after an intravitreal injection of bevacizumab for advanced retinopathy of prematurity. Graefes Arch Clin Exp Ophthalmol 2008;246:1061-1063.

13 Bakri SJ, Snyder MR, Reid JM, Pulido JS, Singh RJ: Pharmacokinetics of intravitreal bevacizumab (Avastin). Ophthalmol 2007;114:855-859.

14 Bakri SJ, Snyder MR, Reid JM, Pulido JS, Ezzat MK, Singh RJ: Pharmacokinetics of intravitreal ranibizumab (Lucentis). Ophthalmol 2007;114:2179-2182.

15 Avery RL: Bevacizumab (Avastin) for retinopathy of prematurity: wrong dose, wrong drug, or b oth? J AAPOS 2012;16:2-4. 